\section{Identifying factors associated with increased mortality risk in stage I NSCLC}

Of all patients diagnosed with lung cancer, only around $25 \%$ are likely to benefit from curative surgical resection-those with stage I or stage II disease. Ignatius Ou et al. carried out a population-based analysis of patients with stage I non-small-cell lung cancer (NSCLC) to identify features associated with increased risk of mortality.

The study analyzed data from the California Cancer Registry from 1989 to 2003, identifying 19,702 patients with stage I NSCLC. The most common age at diagnosis was $70-74$ years. Several characteristics were shown to reduce the survival rate, including male sex, advanced age at diagnosis, low socioeconomic status, no surgical intervention and poorly differentiated histologic grade (stage IA NSCLC: hazard ratio 1.13, 95\% Cl 1.08-1.19; stage IB NSCLC: hazard ratio 1.11, 95\% Cl, 1.07-1.16). Tumors located outside the upper lobe and $\geq 4 \mathrm{~cm}$ in size also increased the risk of mortality for stage IB disease. The authors suggest that these factors should be considered during adjuvant trials. Further analysis of these features could help identify high-risk patients who may benefit from adjuvant chemotherapy.

Original article Ignatius Ou S-H et al. (2007) Prognostic factors for survival of stage I nonsmall cell lung cancer patients: a population-based analysis of 19,702 stage I patients in the California Cancer Registry from 1989 to 2003. Cancer 110: $1532-1541$

\section{Radiotherapy increases risk of secondary cancer in patients with cervical cancer}

A serious consequence of radiotherapy among survivors of cervical cancer is the occurrence of a second cancer. High-dose-rate intracavitary brachytherapy (HDR-ICBT) is extensively used in Japan for the management of patients with cervical cancer; however, little is known about the risk of secondary cancer in patients treated with this type of radiotherapy. Ohno et al. evaluated the risk of secondary malignancies in Japanese women who received radiotherapy for cervical carcinoma.

The study included 2,167 patients who underwent HDR-ICBT (82\%) or low-dose-rate intracavitary brachytherapy (12\%) during the period 1961-1986. The total person-years of follow-up was 25,665 and only $2.8 \%$ of the patients were lost to follow-up. Overall, $49 \%$ of patients survived for more than 10 years, while $33 \%$ and $11 \%$ survived for more than 20 and more than 30 years, respectively. In total, 210 secondary cancers were observed and the cumulative risk of secondary cancer at 30 years after radiotherapy was $23.8 \%$. The relative risk and absolute excess risk of second malignancy were 1.2 and 15.8 per 10,000 personyears, respectively, while the excess risk per person per decade of follow-up was $1.6 \%$. The impact of smoking on the risk of secondary cancer was evaluated in 1,031 patients. The relative risk of second malignancies for patients without a smoking history was 1.4, compared with 1.6 for patients with a history of smoking.

The authors conclude that the survival benefit associated with radiotherapy outweighs the risk of secondary malignancies in patients with cervical cancer.

Original article Ohno T et al. (2007) Long-term survival and risk of second cancers after radiotherapy for cervical cancer. Int J Radiat Oncol Biol Phys 69: 740-745

\section{Plasma HER2 and EGFR levels are prognostic factors in patients with prostate cancer}

Studies have shown that HER2 and EGFR expression are associated with disease progression in patients with metastatic or hormonerefractory prostate cancer. A study by Shariat et al. has examined whether the circulating levels of HER2 and EGFR are prognostic in patients with prostate cancer.

The study included 227 patients with clinically localized prostate cancer who were treated with radical prostatectomy and bilateral lymphadenectomy, and had not received any prior hormonal or radiation therapy. The median preoperative HER2 and EGFR levels were $10.0 \mathrm{ng} / \mathrm{ml}$ and $31.4 \mathrm{ng} / \mathrm{ml}$, respectively. Plasma HER2 levels were significantly elevated in patients with seminal vesicle invasion $(P=0.033)$ and high pathologic Gleason sum $(P=0.028)$. Multivariate Cox regression analyses adjusted for standard preoperative predictors showed that high HER2, low EGFR 